\title{
An Efficient Polyvinylidene Fluoride (PVDF) Nanogenerator for Energy Harvesting in Low Frequency Range
}

\author{
Ehsan Ghafari ${ }^{1}$, Tommy Nantung ${ }^{4}$ and $\mathrm{Na} \mathrm{Lu}^{1,2,3^{*}}$
}

There are abundant mechanical energy available in low frequency range, which can be directly converted into electricity using piezoelectric energy harvester. However, very few piezoelectric energy harvester have high conversion efficiency at low frequency range. This study aims to develop an efficient piezoelectric nanogenerator which can be used in low frequency range for energy harvesting applications using PVDF polymers. The feasibility of using PVDF device for energy harvesting was assessed by using a mechanical vibration setup. In addition, the effect of both amplitude and frequency on the voltage output of the PVDF energy harvester has been studied. According to the results, the optimized frequency range for the device was found to be $45 \mathrm{~Hz}$. The results indicated that the voltage output starts to decay at a higher frequency which can be due to the insufficient time for the PVDF nanofiber to be recovered from the induced strain. The variation of the amplitude has a great influence on the voltage output of the piezoelectric device. The voltage output of the PVDF device is enhanced with increasing the amplitude due to the higher amount of induced strain. In fact, the amount of induced strain is the primary source of the available mechanical energy which can be fed into the piezoelectric device to be converted to the electrical energy. The results clearly show that both frequency and amplitude can affect the voltage output of the piezoelectric device. The highest obtained voltage output can be obtained at the frequency range between $30-45 \mathrm{~Hz}$.

Keywords: PVDF; Energy harvesting; Low frequency; Nanofiber

Received 5 August 2019, Accepted 21 September 2019

DOI: $10.30919 / \mathrm{esmm} 5 \mathrm{f} 321$

\section{Introduction}

Recent studies on piezoelectric materials have resulted in the development of a wide variety of piezoelectric devices such as nanogenerators and sensors. Piezoelectric materials have the ability to produce electrical energy from mechanical energy; for example, they can convert mechanical behavior like vibrations into electricity. This effect is called "direct effect" in which the electric charge develops as a result of the mechanical stress. This effect has been applied to develop several devices such as energy harvester which can be used in applications where outside power is unavailable, and batteries are not a feasible option. The energy harvester nanogenerator can also be used as an individual power source for small electronic devices. ${ }^{1-12}$ In civil engineering, this can be applied to power the wireless sensor for health monitoring of the bridges. Among common piezoelectric materials, the $\beta$-phase of PVDF is one of promising candidates for energy harvesting application in civil infrastructures due to its high piezoelectric properties and flexibility. It has been reported that the sensitivity of PVDF sensors is related to the content of $\beta$-phase so that the higher $\beta$-phase will result

${ }^{1}$ Lyles School of Civil Engineering, Sustainable Materials and Renewable Technology (SMART) Lab, Purdue University, U.S.A.

${ }^{2}$ School of Materials Engineering, Purdue University

${ }^{3}$ Birck Nanotechnology Center, Purdue University

${ }^{4}$ Office of Research and Development, Indiana Department of Transportation

*E-mail: luna@purdue.edu in a better sensitivity of the sensor. ${ }^{13,14}$ Hence, the enhancement of $\beta$ phase of PVDF is a critical step for making an efficient PVDF piezoelectric sensor. In spite of several efforts to improve the $\beta$-phase content, it is still a challenge to fabricate a PVDF sensor with high efficiency due to the complication of the required post-treatment process which mainly includes electrical poling and mechanical stretching. These processes are costly and time-consuming which limit the scalable production of nanogenerators/sensor in the industrial fabrication process. Nevertheless, the mechanical vibrations can be very large in many cases such as civil infrastructure applications; e.g. pavement, the cable-stayed bridges, skyscrapers, railway tracks, etc. An efficient and scalable piezoelectric device is therefore needed which can be either used as an energy harvester to capture the mechanical vibration or as a robust sensor to monitor the structural behaviors in civil infrastructure applications. The viability of using PVDF to produce electrical energy from mechanical energy has been widly reported. ${ }^{15-21}$ However, the development of PVDF nanogenerator for energy harvesting in low frequency range has not been fully explored. In addition, the effect of different mechanical vibration parameters such as frequency and amplitude on the voltage output of PVDF energy harvester has not been well understood. To this end, this study aims to develop an efficient piezoelectric nanofiber device which can be used in energy harvesting in low frequency range applications. The electrospinning method is used for the synthesis of $\beta$-phase of PVDF piezoelectric nanofiber composite. The scalability and low cost of this production method make it considerably attractive for producing piezoelectric nanocomposites for industrial and civil engineering applications. In addition, the effect of both amplitude and frequency on the voltae output of the as-syntheszied 
PVDF energy harvester has been studied.

\section{Experimental study}

\subsection{PVDF synthesize}

The PVDF nanofiber was fabricated using the sol-gel process. PVDF pellet $(\mathrm{Mw}=275000), \mathrm{N}, \mathrm{N}$-dimethylformamide (DMF, Sigma 99.5\%) and acetone (Sigma, 99.7\%) were purchased from Sigma-Aldrich. The PVDF solution was prepared by dissolving PVDF pellets in solvent mixtures of DMF/Acetone. The solution was heated at $70{ }^{\circ} \mathrm{C}$ for 1 -hour following by 5 hours stirring at room temperature. The homogenous PVDF solution was then added to the 10-ml plastic syringe which was placed in a syringe pump. The positive voltage of $15 \mathrm{kV}$ was applied to the needle to form Tylor cone. The nanofibers were spawned on a grounded rotating drum collector, which was placed at a distance of 10 $\mathrm{cm}$ from the tip of the needle.

\subsection{Nanogenerator Fabrication}

The interdigitated electrode device (IDE) has been widely used for fabrication of sensors and energy harvester. ${ }^{22}$ The electrode pattern in IDE device provides an opportunity for utilizing the piezoelectric $d_{33}$ mode, which leads to higher power output. ${ }^{23}$ Moreover, the electrode pattern on IDE-based can be optimized to match the frequency of the mechanical vibration. ${ }^{24}$ In this study, an IDE-device is fabricated using lithography process and inkjet printing method. Device fabrication using lithography process is based on conventional lift-off procedure as shown in Fig. 1. The Kapton and PET were used as substrates to make the flexible device. The fabrication process of the flexible device using lithography method involved several steps. The flexible substrate was first chemically cleaned for the removal of any residual matter from the surface of the substrate. The cleaning process involves soaking the substrate in toluene, acetone, isopropanol and for 5 minutes each and rinsed with DI water at the end. The substrate was then dried with nitrogen gas. A photoresist (PR) material was spun on the flexible substrate using a spin coater in three consecutive steps: 1) 1s ramp to $500 \mathrm{rpm}$, dwell $10 \mathrm{~s}$; 2) $2 \mathrm{~s}$ ramp to $2000 \mathrm{rpm}$, dwell $40 \mathrm{~s}$; 3) 2s ramp down to $0 \mathrm{rpm}$. The substrate was then baked at $90{ }^{\circ} \mathrm{C}$ for ten minutes. The mask alignment systems allowed accurate alignment of substrates with a mask and exposed them to ultraviolet radiation to transfer the pattern of the mask to the substrate for further processing to produce an interdigitated device. The e-beam evaporation was used to deposit a 10$\mathrm{nm}$ chromium film layer as an adhesion layer followed by deposition of a $90 \mathrm{~nm}$ of the gold layer as an electrode. The PR was then removed by
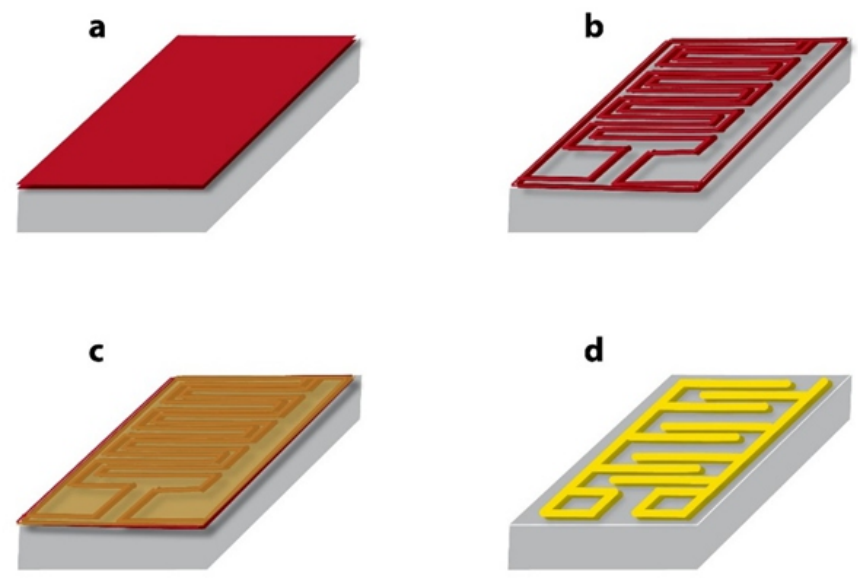

Fig. 1 The fabrication process of interdigitated electrode device; a) The PR spin coating b) Mask alignment c) Deposition of Ti/gold d) Lift-off.

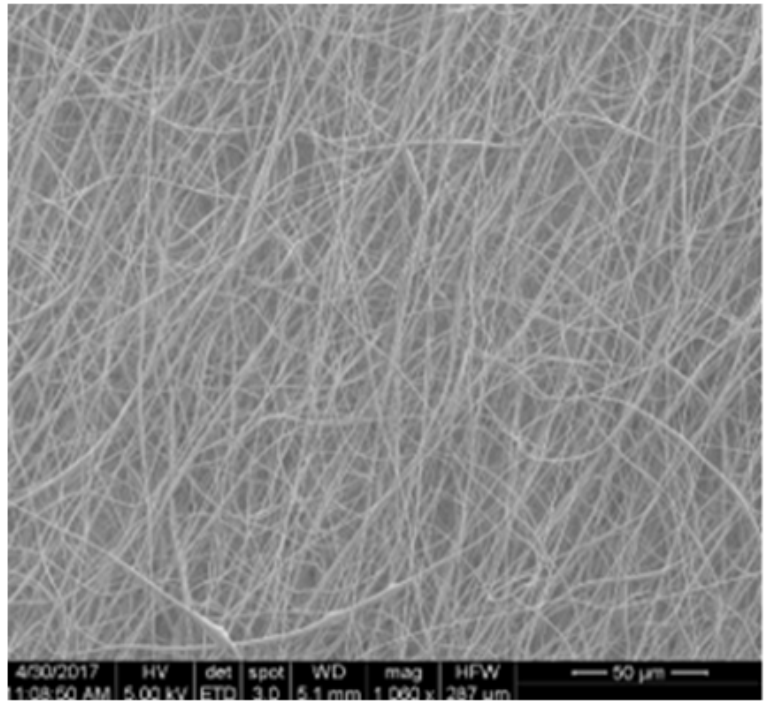

Fig. 2 The SEM images of PVDF nanofiber samples. 
soaking the samples in acetone bath under the fume hood for 14 hours. The PR was removed successfully, and the IDE electrode pattern was formed on the flexible substrate. After that, the lift-off process defines the electrode area, and electrospinning was performed to deposit PVDF nanofibers. A TL-Pro-BM electrospinning machine was used to fabricate the PVDF nanofibers. Fig. 2 shows the SEM imgae of electrospun PVDF nanofiber. Finally, a PDMS layer was spun on the device to protect the device.

\section{Results and discussion}

The piezoelectric energy harvester converts the mechanical energy to electrical which can be used as a sustainable source for different electronic devices such as sensors or wireless sensor network. In fact, the mechanical vibration can be used as a free source to power the wireless sensor which leads to a reduction in the maintenance costs for battery replacement and also the chemical waste of conventional batteries. When the PVDF piezoelectric device is bent, compressive stress is applied on the nanogenerator. This stress will generate a piezoelectric potential due to the direct piezoelectric effect. In order to balance the piezoelectric potential, the free electron moves from low potential to high potential side and accumulate at the surface of the electrodes. When the pressure is removed, the free electron will move back and a voltage signal pulse associated with an opposite pulse voltage output. In order to study the energy harvesting ability of our device, the voltage signal output of nanogenerator was recorded in two steps. At first step, the nanogenerator was not subjected to the load, and the output voltage shows zero voltage output as shown in Fig. 3. At the second step, the nanogenerator was subjected to a compression force, and the positive and negative signal output was observed as shown in Fig. 4. The positive sign was attributed to the compressive stress which causes the PVDF nanofiber membrane to deform, while the negative sign derived from the remove of the compressive force and subsequently induced strain. Therefore, by applying and removing the load in each cycle, the positive and negative voltage signals were observed. It is important to mention that no external load resistor was used in this study. An energy harvesting performance set-up was also developed to assess the efficiency of the nanogenerator voltage output at the different frequencies. The PVDF nanofiber membrane was firmly clamped between two plates to simulate the cantilever beam behavior. In order to evaluate their strain-sensing performance, an electromechanical generator excited from $20 \mathrm{~Hz}$ to $100 \mathrm{~Hz}$ by a function generator which apply periodic force on the cantilever beam.

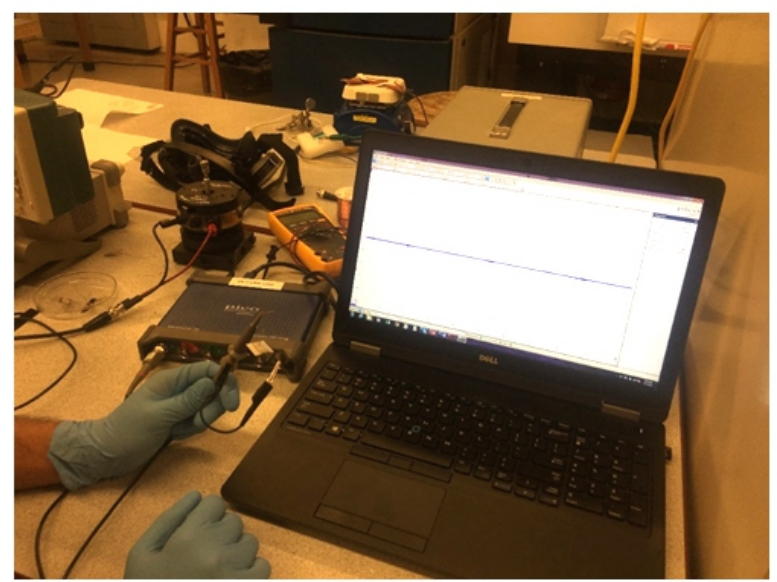

Fig. 3 Unloaded nanogenerator and no signal output.

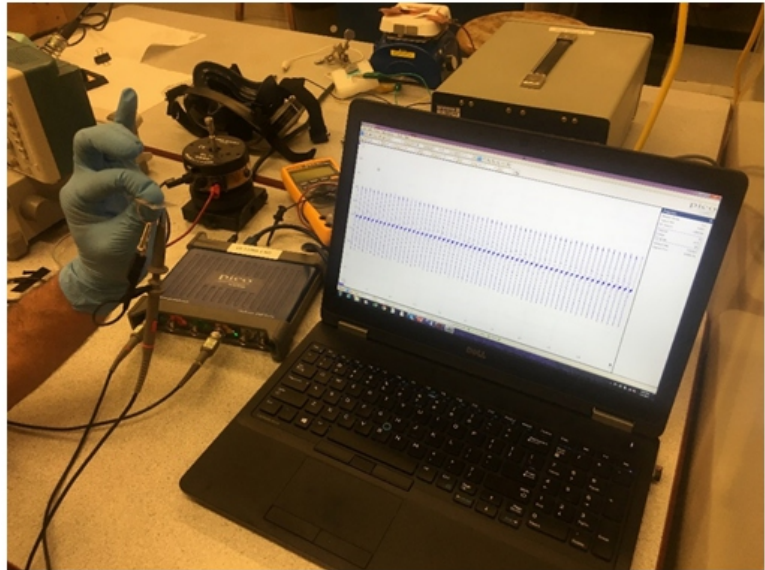

Fig. 3 Unloaded nanogenerator and no signal output.

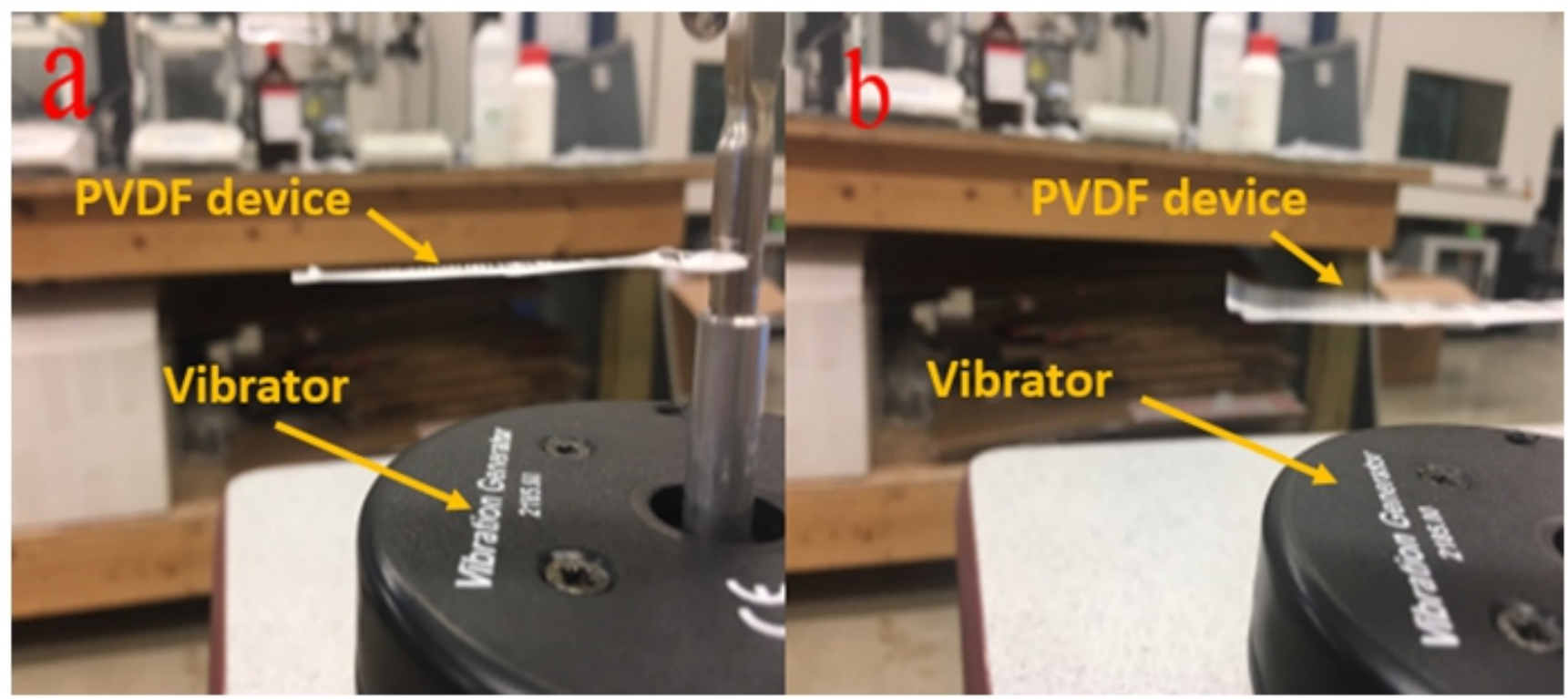

Fig. 5 An energy harvester set up to evaluate the energy harvesting performance of the PVDF nanogenerator in (a) inactive (a) and (b) active condition. 
Fig. 5 shows the energy harvester set up to evaluate the energy harvesting performance of the PVDF nanogenerator in both inactive and active condition. An oscilloscope was also used to record the voltage output of the piezoelectric device. The main problem in energy harvesting in civil infrastructure is the mismatch between the intrinsic frequencies of harvesters and the resonant frequencies of the civil infrastructure. It is very difficult to match the resonant frequencies of the energy harvesters with the mechanical vibration frequencies due to the high stiffness and brittleness of piezoelectric patches. Generally, the ambient vibration frequencies in civil infrastructure which are usually lower than $30 \mathrm{~Hz}$. Moreover, even if the resonant frequency can be tuned as low as required, the limitation of small strain allowed in piezoelectric patches will lead to a low energy output power. Fig. 6 shows a typical voltage output of nanofiber device with an active area of $2.25 \mathrm{~cm}^{2}$ at the frequency of $30 \mathrm{~Hz}$. As can be seen, the PVDF nanogenerators exhibit a maximum peak output voltage of $450 \mathrm{mV}$ at the frequency of $30 \mathrm{~Hz}$. In general, the amplitude and the frequency are two important parameters of the AC signal wave which characterize the properties of AC signal. The frequency of AC signal is the rate of changing direction and is determined based on the number of forwards/ backward cycles per second, while the amplitude is defined as the maximum voltage achieved by the AC signal. The applied mechanical vibration generates the $\mathrm{AC}$ waveform which can excite the piezoelectric device to generate power. In fact, the properties of AC excitation signal will affect the performance of the piezoelectric device and, as a result, the voltage output. Therefore, the study of the AC signal's parameters is of great importance. With this in mind, it is important to study the effect of the frequency and amplitude of the AC signal on the voltage output of the PVDF nanofiber piezoelectric device. The samples were encapsulated by a PDMS protective layer. The frequency range was varied from $20 \mathrm{~Hz}$ up to $100 \mathrm{~Hz}$, while the mechanical vibration set-up was excited with an AC signal with varying amplitude. The amplitude voltage is reported as "peak to peak" (Vpp) which indicates the difference between the maximum positive and the maximum negative amplitudes of a waveform. The AC signal was generated with amplitude from $3 \mathrm{Vpp}$ to $20 \mathrm{Vpp}$. Fig. 7 shows the variation of the voltage output with different frequency at the fixed amplitude of $5 \mathrm{Vpp}$ on the voltage output of the piezoelectric device. As can be seen, the frequency variation exhibited a significant effect on the voltage output. The frequency of the signal was increased up to $100 \mathrm{~Hz}$ in $5 \mathrm{~Hz}$ increments, while the voltage output was recorded at each step using an oscilloscope. The results indicated that the obtained voltage output at the frequency of $20 \mathrm{~Hz}$ is $109 \mathrm{mV}$. As can be seen, the voltage output of the PVDF device is enhanced when the frequency increased. The highest

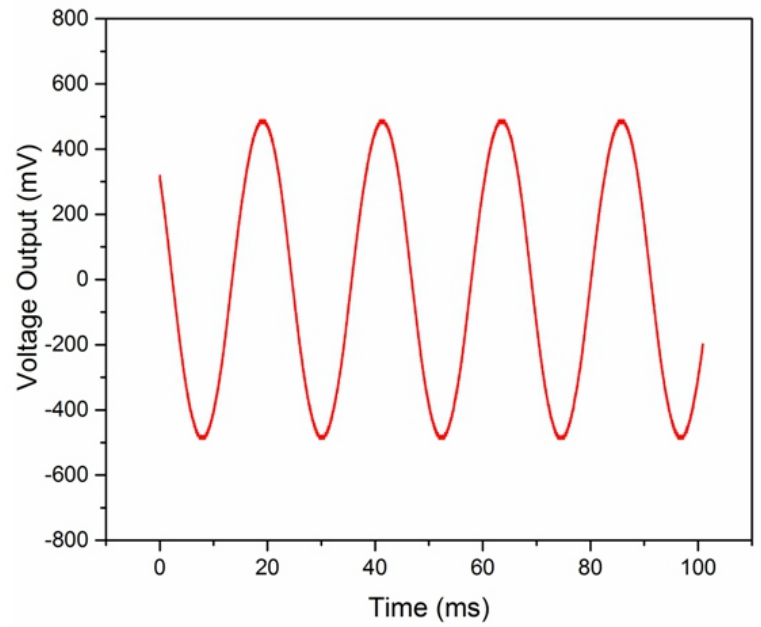

Fig. 6 Voltage output measurement by oscilloscope at the frequency of $30 \mathrm{~Hz}$.

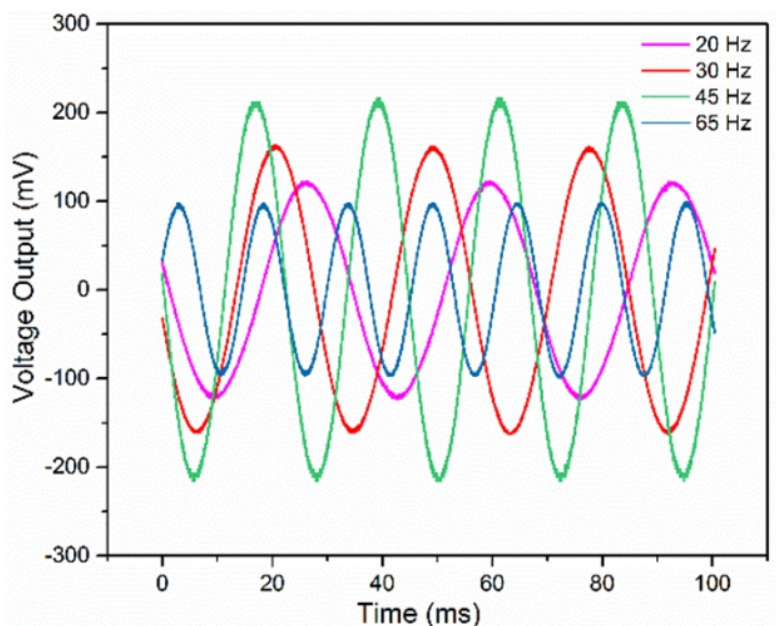

Fig. 7 The voltage output at AC signal with amplitude of 5 Vpp versus the frequency ranges.

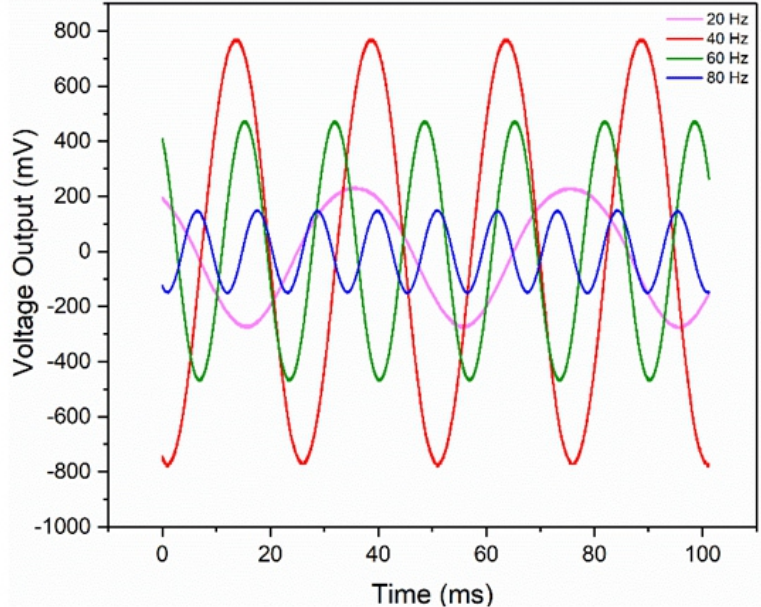

Fig. 8 The voltage output at AC signal with an amplitude of $20 \mathrm{Vpp}$ versus the frequency ranges.

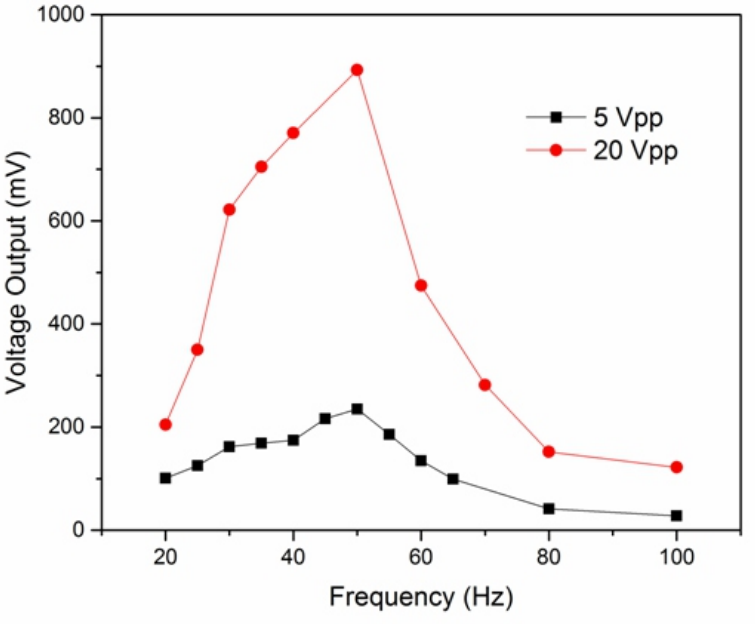

Fig. 9 The maximum voltage output at AC signal with different amplitude. 
voltage peak was obtained at the frequency of $45 \mathrm{~Hz}$. As shown, the voltage output became $200 \mathrm{mV}$ when the frequency was increased up to $45 \mathrm{~Hz}$.

This experiment was repeated to determine the effect of the frequency variation on the voltage output at a higher amplitude. The amplitude of the signal as increased to $20 \mathrm{Vpp}$. Fig. 8 presents the voltage output of the piezoelectric device as a function of the frequency at fixed $20 \mathrm{Vpp}$ amplitude. In general, the variation of the frequency with the AC amplitude of $20 \mathrm{Vpp}$ exhibited the same trend as the AC amplitude of $5 \mathrm{Vpp}$. However, as can be seen, the effect of the frequency variation on the voltage output is more pronounced when the AC signal is applied with higher amplitude. For example, the highest peak of the voltage output is $850 \mathrm{mV}$ with the AC amplitude of $20 \mathrm{Vpp}$, which is almost 7 times higher than the voltage output of the device with the AC amplitude of $5 \mathrm{Vpp}$. According to the results, the optimized frequency range for the device was found to be $45 \mathrm{~Hz}$. However, the voltage output starts to decrease with further increase of the frequency. Fig. 9 presents the maximum obtained voltage output as a function of frequency at both amplitudes of $5 \mathrm{Vpp}$ and $20 \mathrm{Vpp}$. As can be seen, the variation of the amplitude has a great influence on the voltage output of the piezoelectric device. Both curves show the maximum voltage output at $45 \mathrm{~Hz}$ regardless of the amplitude range. The results indicated that the voltage output starts to decay at a higher frequency which can be due to the insufficient time for the PVDF nanofiber to be recovered from the induced strain. The results imply the efficiency of the developed piezoelectric device for low-frequency cases. To analyze the effect of the amplitude the frequency of the AC signal was fixed at $45 \mathrm{~Hz}$, and the amplitude varied from $3 \mathrm{Vpp}$ to $20 \mathrm{Vpp}$. Fig. 10 shows the variation of the voltage output of the piezoelectric device for the amplitude of 5 Vpp, $10 \mathrm{Vpp}, 15 \mathrm{Vpp}$ and $20 \mathrm{Vpp}$. The highest peak of the voltage output was plotted versus the amplitude in Fig. 11. As shown, a perfect linear relation can be observed between the amplitude and the voltage output.

The voltage output is increasing with increasing the amplitude. One possible attribute for this result is that the higher amplitude AC signal induces higher strain on PVDF samples which leads to higher

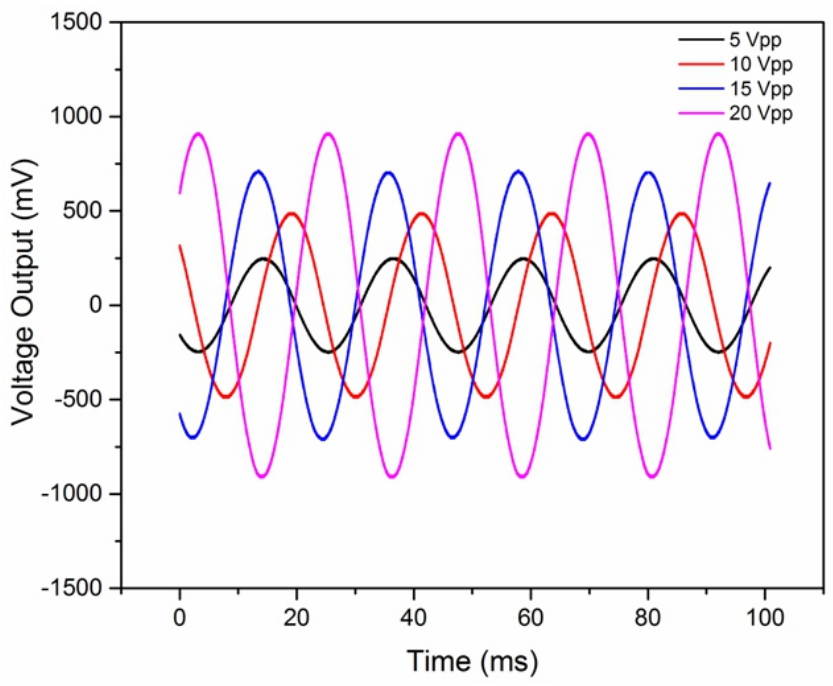

Fig. 10 The maximum voltage output at AC signal with a different amplitude at the fixed frequency of $45 \mathrm{~Hz}$.

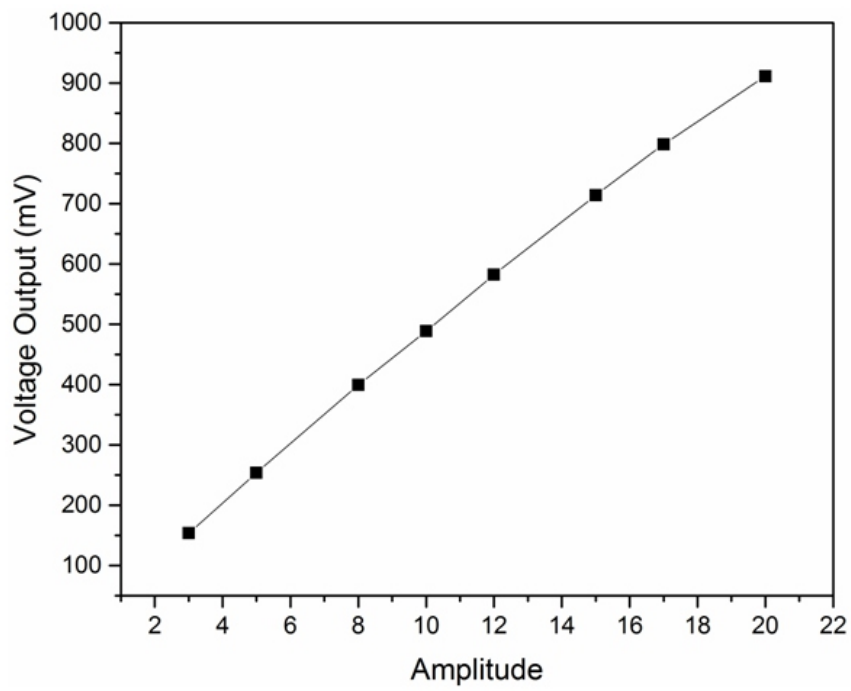

Fig. 11 The maximum voltage output at AC signal with different amplitude at the fixed frequency of $45 \mathrm{~Hz}$.

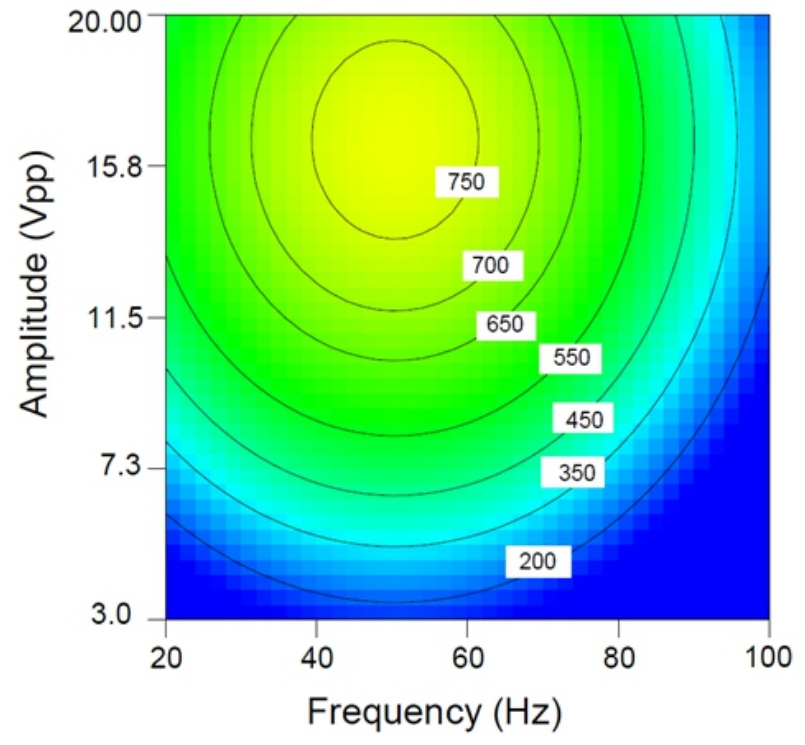

Fig. 12 Two-dimensional counter plot showing the interaction effect of the amplitude and frequency of the AC signal on the voltage output. 
charge separation and voltage output according to the following equation:

$$
V_{\text {output }}=d_{33} E s T / \varepsilon^{T}
$$

where $\varepsilon^{T}$ is permittivity under a constant strain, $\mathrm{d}_{33}$ is the piezoelectric coefficient, $\mathrm{s}$ is the amount of induced strain, $\mathrm{E}$ is Young's modulus and, $\mathrm{T}$ is the thickness of the film.

Since Young's modulus, thickness and voltage coefficient are the same for the piezoelectric device in this experiment. Therefore the strain can be considered as the only parameter which can affect the voltage output. In fact, the amount of induced strain can be considered as the main source of the available mechanical energy which can be fed into the piezoelectric device to be converted to the electrical energy. The results clearly suggested that both frequency and amplitude can affect the voltage output of the piezoelectric device. Therefore, an interaction effect of these two parameters has been analyzed to have a better understanding of the effect. Fig. 12 shows the two-dimensional counter plot which shows the interaction effect of the amplitude and frequency of the AC signal on the voltage output. The highest voltage output can be obtained by highest amplitude and the frequency range between 30 $45 \mathrm{~Hz}$. This range of frequency has been reported as a typical frequency of the bridges. ${ }^{25}$ The obtained voltage output proved the feasibility of the electrospun PVDF nanofiber to convert the mechanical vibration to the electricity at low frequency regime.

\section{Conclusion}

The feasibility of using PVDF device for energy harvesting was assessed by conducting a series of experiments. According to the results, the optimized frequency range for the device was found to be $45 \mathrm{~Hz}$. The results indicated that the voltage output starts to decay at a higher frequency which can be due to the insufficient time for the PVDF nanofiber to be recovered from the induced strain. The variation of the amplitude has a great influence on the voltage output of the piezoelectric device. The voltage output of the PVDF device is enhanced with increasing the amplitude due to the higher amount of induced strain. In fact, the amount of induced strain can be considered as the main source of the available mechanical energy which can be fed into the piezoelectric device to be converted to the electrical energy. The results clearly show that both frequency and amplitude can affect the voltage output of the piezoelectric device. The highest obtained voltage output can be obtained at the frequency range between $30-45 \mathrm{~Hz}$. The obtained voltage output generated in a low-frequency range is very beneficial in harvesting electrical energy from the existing mechanical vibration in civil infrastructure. The results can assist in adopting the electrospun nanofiber device in energy harvesting application in infrastructure such as bridge deck and highway pavement.

\section{Acknowledgment}

The authors at Purdue University are grateful to the funding supports from Indiana Department of Transportation (SPR-4210).

\section{References}

1. Z. L. Wang, G. Zhu, Y. Yang, S. Wang and C. Pan, Mater. Today, 2012, 15 532-543

2. C. Zhou, A. Ghods, K. Yunghans, V. Saravade, P. Patel, X. Jiang, B. Kucukgok, N. Lu and I. Ferguson, in Oxide-based Materials and Devices VIII. 2017.

3. E. Ghafari, X. Jiang and N. Lu, Adv. Compos. Hybrid Mater., 2017, 1, 332-340.

4. Z. Liu, X. Yi, J. Wang, J. Kang, A. Melton, Y. Shi, N. Lu, J. Wang, J. Li, and I. Ferguson, Appl. Phys. Lett., 2012, 100, 232408.

5. Z. Liu, X. Yi, Z. Yu, G. Yuan, Y. Liu, J. Wang, J. Li, N. Lu, I. Ferguson, and Y. Zhang, Sci. Rep-UK, 2016, 6, 23950.

6. B. Hussain, M. Raja, N. Lu and I. Ferguson, in High Capacity Optical Networks and Enabling Technologies (HONET-CNS), 2013 10th International Conference on. 2013. IEEE.

7. B. Kucukgok, B. Wang, A. G. Melton, N. Lu and I. Ferguson, Phys. Status Solidi C, 2014, 11, 894-897.

8. B. Hussain, B. Kucukgok, M. Y. A. Raja, B. Klein, N. Lu and I. Ferguson, Oxide-based Materials and Devices V, 2014, 8987.

9. Y. Feng, X. Jiang, E. Ghafari, B. Kucukgok, C. Zhang, I. Ferguson and N. Lu, Adv. Compos. Hybrid Mater, 2018, 1, 114-126.

10. E. Ghafari, S. A. Ghahari, Y. Feng, F. Severgnini and N. Lu, Compos. Part BEng, 2016, 105, 160-166.

11. E. Ghafari, F. Severgnini, S. Ghahari, Y. Feng, E. J. Lee, C. Zhang, X. Jiang, N. Lu, Z. Guo and Y. Chen, Multifunctional Nanocomposites for Energy and Environmental Applications, 2018, 173-202.

12. E. Ghafari, E. Witkoske, Y. Liu, C. Zhang, X. Jiang, A. Bukowski, B. Kucukgok, M. Lundstrom, I.Ferguson and N. Lu, III-Nitride Materials, Devices and Nano-Structures, 2017, 37.

13. B. Li, C. Xu, J. Zheng and C. Xu, Sensors, 2014, 14, 9889-9899.

14. M. Hosseini and A. S. H. Makhlouf, Industrial Applications for Intelligent Polymers and Coatings, Springer, 2016.

15. H. Yu, T. Huang, M. Lu, M. Mao, Q. Zhang and H. Wang, Nanotechnology, 2013, 24, 405401.

16. V. Bhavanasi, V. Kumar, K. Parida, J. Wang and P. S. Lee, ACS Appl. Mater. Inter., 2015, 8, 521-529.

17. Z. Liu, C. T. Pan, L. W. Lin, H. W. Li, C. A. Ke, J. C. Huang, P. S. Wang, in Nano/Micro Engineered and Molecular Systems (NEMS), 2013 8th IEEE International Conference on. 2013. IEEE.

18. S. Tiwari, A. Gaur, C. Kumar and P. Maiti, Energy, 2019, 171, 485-492.

19. M. Abbasipour, R. Khajavi, A. A. Yousefi, M. E. Yazdanshenas, F. Razaghian and A. Akbarzadeh, Polym. Adv. Technolo., 2019, 30, 279-291.

20. D. N. Nguyen and W. Moon, J. Sens. Sci. Technolo., 2019, 28, 205-215.

21. S. You, L. Zhang, J. Gui, H. Cui and S. Guo, Micromachines, 2019, 10, 302.

22. W. H. Duan, Q. Wang and S. T. Quek, Materials, 2010, 3, 5169-5194.

23. C. Lee, D. Wood, D. Edmondson, D. Yao, A. E. Erickson, C. T. Tsao, R. A. Revia, H. Kim and M. Zhang, Ceram. Int., 2016, 42, 2734-2740.

24. H. Gu, G. M. Lloyd and M. L. Wang, in Smart Structures and Materials. 2005. International Society for Optics and Photonics.

25. M. Peigney and D. Siegert, Smart Mater. Struct., 2013, 22, 095019.

Publisher's Note Engineered Science Publisher remains neutral with regard to jurisdictional claims in published maps and institutional affiliations. 